\title{
A first-prototype multi-determinant $X$-ray constrained wavefunction approach: the $X$-ray constrained extremely localized molecular orbital-valence bond method
}

\section{Alessandro Genoni}

Acta Cryst. (2017). A73, 312-316

\section{- IUCr Journals CRYSTALLOGRAPHY JOURNALS ONLINE \\ Copyright (C) International Union of Crystallography \\ Author(s) of this paper may load this reprint on their own web site or institutional repository provided that this cover page is retained. Republication of this article or its storage in electronic databases other than as specified above is not permitted without prior permission in writing from the IUCr. \\ For further information see http://journals.iucr.org/services/authorrights.html}




\section{short communications}

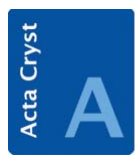

FOUNDATIONS

ADVANCES

ISSN 2053-2733

Received 3 March 2017

Accepted 19 April 2017

Edited by J.-G. Eon, Universidade Federal do Rio de Janeiro, Brazil

Keywords: X-ray constrained wavefunction approach; extremely localized molecular orbitals; resonance structures; valence-bond method.

Supporting information: this article has supporting information at journals.iucr.org/a

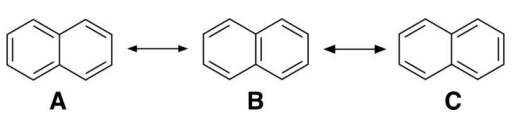

(C) 2017 International Union of Crystallography

\section{A first-prototype multi-determinant X-ray constrained wavefunction approach: the X-ray constrained extremely localized molecular orbital- valence bond method}

\author{
Alessandro Genoni*
}

CNRS, Laboratoire SRSMC, UMR 7565, 1 Boulevard Arago, Metz, F-57078, France, and Université de Lorraine, Laboratoire SRSMC, UMR 7565, 1 Boulevard Arago, Metz, F-57078, France. *Correspondence e-mail:

alessandro.genoni@univ-lorraine.fr

All the current variants of Jayatilaka's X-ray constrained wavefunction (XCW) approach work within the framework of the single-determinant wavefunction ansatz. In this paper, a first-prototype multi-determinant XCW technique is proposed. The strategy assumes that the desired XCW is written as a valencebond-like expansion in terms of pre-determined single Slater determinants constructed with extremely localized molecular orbitals. The method, which can be particularly suitable to investigate systems with a multi-reference character, has been applied to determine the weights of the resonance structures of naphthalene at different temperatures by exploiting experimental highresolution X-ray diffraction data. The results obtained have shown that the explicit consideration of experimental structure factors in the determination of the resonance structure weights may lead to results significantly different compared with those resulting only from the simple energy minimization.

\section{Introduction}

The possibility of extracting wavefunctions or density matrices from experimental crystallographic data has been a tempting prospect for a long time (Grabowsky et al., 2017). In this context, a prominent role is occupied by the strategies originally proposed by Clinton, Massa and co-workers in the 1960s and later (Clinton, Galli \& Massa, 1969; Clinton, Nakhleh \& Wunderlich, 1969; Clinton, Henderson \& Prestia, 1969; Clinton, Galli, Henderson et al., 1969; Clinton \& Lamers, 1969; Clinton \& Massa, 1972a). They initially used only theoretically generated X-ray diffraction data as external constraints (Clinton \& Massa, 1972b; Clinton et al., 1973; Frishberg \& Massa, 1978, 1981, 1982; Goldberg \& Massa, 1983; Boehme \& La Placa, 1987) and the very first attempt at exploiting real experimental structure factors was performed in 1985 (Massa et al., 1985). Since then, inspired by the pioneering techniques of Clinton and Massa, several researchers have proposed different strategies to obtain wavefunctions or density matrices compatible with experimental diffraction data (Grabowsky et al., 2017), not exclusively X-ray structure factors. Among them, it is worth citing the methods aimed at reconstructing diagonal and offdiagonal parts of one-electron density matrices by exploiting simultaneously X-ray diffraction and inelastic Compton scattering measurements, such as those proposed by Weyrich and collaborators (Schmider et al., 1990, 1992; Weyrich, 2006) or 
those developed by Gillet, Becker and Cortona (Gillet et al., 2001; Gillet \& Becker, 2004; Gillet, 2007).

However, nowadays, among all the modern experimentally constrained wavefunction or density matrix strategies, the $\mathrm{X}$-ray constrained wavefunction $(\mathrm{XCW})$ approach proposed by Jayatilaka (Jayatilaka, 1998, 2012; Jayatilaka \& Grimwood, 2001; Grimwood \& Jayatilaka, 2001; Bytheway, Grimwood \& Jayatilaka, 2002; Bytheway, Grimwood, Figgis et al., 2002; Grimwood et al., 2003) is the most promising and reliable. The $\mathrm{XCW}$ method basically consists in finding a plausible wavefunction that not only variationally minimizes the energy of the system under examination, but also reproduces a set of experimentally collected structure-factor amplitudes within the limit imposed by the experimental errors. Initially developed in the framework of the restricted Hartree-Fock formalism (Jayatilaka, 1998; Jayatilaka \& Grimwood, 2001; Grimwood et al., 2003), the Jayatilaka strategy has since been extended to the unrestricted case (Hudák et al., 2010) and to relativistic corrections (Hudák et al., 2010; Bučinský et al., 2016). Furthermore, the XCW technique has also been coupled with a method proposed by Stoll et al. (1980) to determine extremely localized molecular orbitals (ELMOs) (Sironi et al., 2007). This led to the novel X-ray constrained ELMO (XC-ELMO) strategy (Genoni, 2013a,b; Dos Santos et al., 2014; Genoni \& Meyer, 2016) which enables extraction of molecular orbitals that are strictly localized on small molecular fragments (e.g. atoms, bonds or functional groups) and compatible with experimental X-ray diffraction data. However, all the XCW methods developed so far are limited to the single Slater determinant wavefunction ansatz and this is probably one of the most plausible reasons why the current $\mathrm{XCW}$ approaches seem to capture electron correlation effects only partially (Genoni et al., 2017).

In this communication, we present a first attempt to go beyond the single-determinant wavefunction ansatz in the framework of the Jayatilaka approach. To accomplish this task we exploited again the concept of ELMOs that enabled us to define the basis of Slater determinants on which to expand the wavefunction to be determined. This gave rise to the new multi-determinant X-ray constrained ELMO valence bond (XC-ELMO-VB) method, which can be particularly useful to treat systems characterized by a multi-reference character and which consequently allows one to extract the weights of the different resonance structures of the investigated molecule from experimental X-ray diffraction data.

\section{Theory}

As in the usual XCW techniques, we assume that we are working with effective molecular crystals whose noninteracting molecular units are described by formally identical and symmetry-related wavefunctions. Furthermore, assuming that each molecular unit is a symmetry-unique portion of the crystal unit cell, we can write the global unit-cell electron density as the sum of $N_{m}$ crystal unit charge distributions $\rho_{k}(\mathbf{r})$, which are simply obtained from the reference electron density $\rho_{0}(\mathbf{r})$ by means of the unit-cell symmetry operations $\left\{\mathbf{Q}_{k}, \mathbf{q}_{k}\right\}$ :

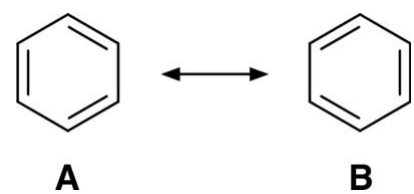

Figure 1

The two resonance structures of benzene can be used as localization schemes for two different ELMO calculations providing two different ELMO wavefunctions.

$$
\rho_{\text {cell }}(\mathbf{r})=\sum_{k=1}^{N_{m}} \rho_{k}(\mathbf{r})=\sum_{k=1}^{N_{m}} \rho_{0}\left[\mathbf{Q}_{k}^{-1}\left(\mathbf{r}-\mathbf{q}_{k}\right)\right] .
$$

To guarantee the exactitude of equation (1), in all the Jayatilaka-type approaches, the electron density $\rho_{0}(\mathbf{r})$ is associated with the wavefunction $\left|\Psi_{0}\right\rangle$ that not only minimizes the electronic energy of the reference unit, but that also reproduces a set of experimental structure-factor amplitudes $\left\{\left|F_{\mathbf{h}}^{\exp }\right|\right\}$. Nevertheless, while in the usual XCW methods $\left|\Psi_{0}\right\rangle$ has the form of a single Slater determinant, in the new XC-ELMO-VB technique we express the reference wavefunction in the following form:

$$
\left|\Psi_{0}\right\rangle=\left|\Psi_{\text {XC-ELMO-VB }}\right\rangle=\sum_{i} C_{i}\left|\Psi_{i}\right\rangle
$$

where the functions $\left\{\left|\Psi_{i}\right\rangle\right\}$ are single Slater determinants that describe all the possible resonance structures of the system under examination. In our case, they will consist of normalized ELMO wavefunctions that are pre-determined by means of unconstrained ELMO calculations that exploit localization schemes corresponding to the different resonance structures (more details about the ELMO method are given in the supporting information). For example, if we were interested in studying benzene, the wavefunction $\left|\Psi_{\mathrm{XC}-\mathrm{ELMO}-\mathrm{VB}}\right\rangle$ would be a linear combination of at least two ELMO wavefunctions: one corresponding to the localization scheme associated with resonance structure $A$ and another one corresponding to the ELMO wavefunction associated with the localization scheme for resonance structure $B$ (see Fig. 1).

In the current version of the technique, the pre-optimized unconstrained ELMOs are kept frozen, while the coefficients $\left\{C_{i}\right\}$ in equation (2) are determined by minimizing the following functional:

$$
J[\mathbf{C}]=E_{\text {ELMO }-\mathrm{VB}}[\mathbf{C}]+\lambda\left(\chi^{2}[\mathbf{C}]-\Delta\right),
$$

where $[\mathbf{C}]$ indicates the functional dependence on the coefficients $\left\{C_{i}\right\}$ of expansion (2), $E_{\mathrm{ELMO}-\mathrm{VB}}$ is the electronic energy associated with $\left|\Psi_{\mathrm{XC}-\mathrm{ELMO}-\mathrm{VB}}\right\rangle, \lambda$ is an external parameter representing the strength of the constraints given by the experimental data, $\Delta$ is the desired agreement between theoretical and experimental values (usually set equal to 1.0) and $\chi^{2}$ is the measure of the fitting accuracy between calculated and observed structure-factor amplitudes, namely

$$
\chi^{2}=\frac{1}{N_{r}-N_{p}} \sum_{\mathbf{h}} \frac{\left(\eta\left|F_{\mathbf{h}}^{\mathrm{calc}}\right|-\left|F_{\mathbf{h}}^{\exp }\right|\right)^{2}}{\sigma_{\mathbf{h}}^{2}} .
$$

$N_{r}$ is the number of considered X-ray diffraction data, $N_{p}$ the number of adjustable parameters, $\mathbf{h}$ the triads of Miller indices 
Table 1

Results of the unconstrained ELMO calculations (basis set cc-pVDZ) performed on the crystallographic structures of naphthalene determined at $100,135,170$ and $205 \mathrm{~K}$.

\begin{tabular}{lll}
\hline $\begin{array}{l}\text { Temperature and } \\
\text { resonance structure }\end{array}$ & ELMO & \\
\cline { 2 - 3 }$\chi^{2}$ & Electronic energy $\left(E_{h}\right)$ \\
$100 \mathrm{~K} A$ & 1.67 & -841.791171 \\
$100 \mathrm{~K} B$ & 3.35 & -841.821438 \\
$100 \mathrm{~K} C$ & 1.67 & -841.791171 \\
$135 \mathrm{~K} A$ & 1.90 & -841.554899 \\
$135 \mathrm{~K} B$ & 3.87 & -841.585315 \\
$135 \mathrm{~K} C$ & 1.90 & -841.554899 \\
$170 \mathrm{~K} A$ & 2.38 & -842.209337 \\
$170 \mathrm{~K} B$ & 4.37 & -842.239801 \\
$170 \mathrm{~K} C$ & 2.38 & -842.209337 \\
$205 \mathrm{~K} A$ & & \\
$205 \mathrm{~K} B$ & 3.16 & -842.070174 \\
$205 \mathrm{~K} C$ & 5.27 & -842.100581 \\
\hline
\end{tabular}

labelling the reflections, $\sigma_{\mathbf{h}}$ the experimental error associated with the observed structure-factor amplitude $\left|F_{\mathbf{h}}^{\exp }\right|$ and $\eta$ a scale factor that is determined in order to minimize $\chi^{2}$. Of course, the summation in equation (4) runs only over the triads of Miller indices associated with observed reflections, the unobserved ones being excluded from the external constraint.

In analogy with the usual XCW methods, in our new XCELMO-VB approach, the external parameter $\lambda$ is iteratively adjusted until convergence of the weights associated with the resonance structures of the system under examination. In this respect, it is worth noting that, due to the non-orthogonality of the Slater determinants $\left\{\left|\Psi_{i}\right\rangle\right\}$ in expansion (2), the coefficients $\left\{C_{i}\right\}$ do not immediately provide the real weights associated with the corresponding resonance structures. These weights are actually given by the Chirgwin-Coulson coefficients (Chirgwin \& Coulson, 1950), which are defined as

$$
K_{i}=\left|C_{i}\right|^{2}+\sum_{j \neq i} C_{i} C_{j} S_{i j}
$$

where $S_{i j}=\left\langle\Psi_{i} \mid \Psi_{j}\right\rangle$ is the overlap between the pre-determined ELMO wavefunctions $\left|\Psi_{i}\right\rangle$ and $\left|\Psi_{j}\right\rangle$. Therefore, in the current version of the strategy, we stopped the X-ray constrained calculations when the largest absolute variation of the Chirgwin-Coulson coefficients between two consecutive $\lambda$ steps is lower than $1.0 \times 10^{-3}$ :

$$
\max \left\{\left|K_{i}^{\lambda}-K_{i}^{\lambda-1}\right|\right\}_{i}<1.0 \times 10^{-3} .
$$

\section{Test calculations}

The new XC-ELMO-VB technique has been tested using the high-resolution X-ray diffraction data collected for naphthalene by Oddershede \& Larsen (2004) at 100, 135, 170 and $205 \mathrm{~K}$.
At first, for each crystal structure determined at the different temperatures, we performed unconstrained ELMO calculations by considering three possible localization schemes, one for each resonance structure depicted in Fig. 2. In other words, for each localization pattern, other than having atomic fragments for the core electrons and bond fragments for all the $\mathrm{C}-\mathrm{C}$ and $\mathrm{C}-\mathrm{H} \sigma$ bonds, we have also considered $\mathrm{C}-\mathrm{C}$ bond fragments for each $\pi$ bond in the resonance structure. The essential results of the unconstrained ELMO calculations (basis set cc-pVDZ) are shown in Table 1.

It is easy to observe that, for each crystallographic structure, the unconstrained ELMO computations provided the same trends. In fact, the ELMO wavefunction associated with resonance structure $B$ is always the one with the lowest energy, while the ELMO wavefunctions corresponding to resonance structures $A$ and $C$ are characterized by higher energies and are always degenerate. However, in spite of the relative energetic stability, it is worth noting that resonance structures $A$ and $C$ always provide a better statistical agreement with the experimental diffraction data (see the $\chi^{2}$ values) than resonance structure $B$. These results seem to show that, although less energetically favourable in the gas phase, resonance structures $A$ and $C$ are probably more compatible with the collected structure-factor amplitudes and are probably predominant in the crystal phase.

As mentioned above, the goal of the preliminary ELMO computations was also to provide the ELMO Slater determinants over which to expand the valence bond wavefunction [see equation (2)] for the following XC-ELMO-VB calculations. Of course, in this case, for each crystallographic structure we used the ELMO wavefunctions corresponding to resonance structures $A, B$ and $C$ of naphthalene (see Fig. 2). Furthermore, for all the XC-ELMO-VB computations, we exploited unit-cell parameters, anisotropic displacement parameters (ADPs) and structure-factor amplitudes deposited with the corresponding crystallographic structures. The main results of the unconstrained ELMO-VB [external multiplier $\lambda$ set equal to 0.0 in functional (3)] and of the X-ray constrained ELMO-VB calculations are shown in Table 2, where we have reported the $\chi^{2}$ statistical agreements and the ChirgwinCoulson weights obtained in the different cases.

For all the unconstrained ELMO-VB computations, which simply consist in simple energy minimizations without considering the effects of the experimental structure-factor amplitudes, resonance structure $B$ is characterized by a greater Chirgwin-Coulson weight compared with the other two structures. This reflects the relative energetic stability resulting from the unconstrained ELMO calculations. On the contrary,

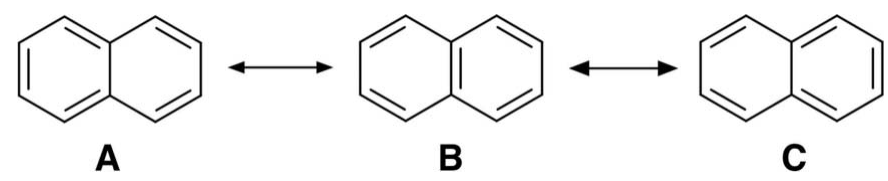

Figure 2

Resonance structures of naphthalene corresponding to the three different localization schemes for the unconstrained ELMO calculations that have been performed. 
Table 2

Statistical agreements with the experimental structure-factor amplitudes and Chirgwin-Coulson weights obtained from the unconstrained $(\lambda=0.0)$ and X-ray constrained ELMO valence bond calculations (basis set cc-pVDZ) performed on the crystallographic structures of naphthalene determined at 100,135, 170 and $205 \mathrm{~K}$.

\begin{tabular}{lllllllllll}
\hline & \multicolumn{3}{l}{ Unconstrained ELMO-VB $(\lambda=0.0)$} & & \multicolumn{6}{c}{ X-ray constrained ELMO-VB } \\
\cline { 2 - 8 } Temperature & $\chi^{2}$ & $K_{A}$ & $K_{B}$ & $K_{C}$ & & $\lambda_{\max }$ & $\chi^{2}$ & $K_{A}$ & $K_{B}$ & $K_{C}$ \\
\hline $100 \mathrm{~K}$ & 1.73 & 0.257 & 0.486 & 0.257 & & 0.39 & 1.51 & 0.365 & 0.270 & 0.365 \\
$135 \mathrm{~K}$ & 2.00 & 0.257 & 0.486 & 0.257 & & 0.38 & 1.76 & 0.367 & 0.265 & 0.367 \\
$170 \mathrm{~K}$ & 2.49 & 0.257 & 0.486 & 0.257 & & 0.38 & 2.25 & 0.367 & 0.266 & 0.367 \\
$205 \mathrm{~K}$ & 3.33 & 0.257 & 0.486 & 0.257 & & 0.36 & 3.10 & 0.362 & 0.276 & 0.362 \\
\hline
\end{tabular}

the atomic ADPs) and can also be seen in Table 1. However, notwithstanding these clear changes in $\chi^{2}$, the ChirgwinCoulson weights remain quite stable (maximal fluctuation lower than 2\%; see Table 2), thus indicating that correct information on the electronic structure of naphthalene can actually be obtained from all the four considered X-ray data sets characterized by

when the experimental diffraction data are directly taken into account in the minimization of functional (3) (XC-ELMO-VB computations), we can observe that resonance structures $A$ and $C$ become predominant (with equal weights) over structure $B$. This confirms what we have previously inferred from the unconstrained ELMO calculations, namely the fact that, although less energetically favourable in the gas phase, resonance structures $A$ and $C$ are more compatible with the collected structure-factor amplitudes than structure $B$, and, therefore, that they become predominant in the crystal phase. Moreover, analysing Table 2, we can also observe that, as expected, the statistical agreement with the experimental X-ray diffraction data improves when lower-temperature experimental data sets are considered in the calculations. This is obviously due to the better deconvolution of thermal motion from the static electron density (i.e. better quality of

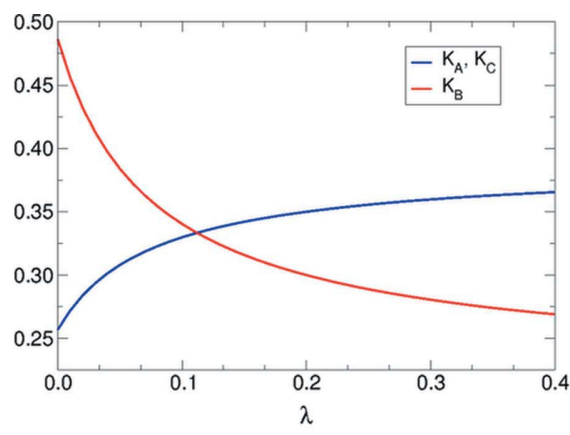

(a)

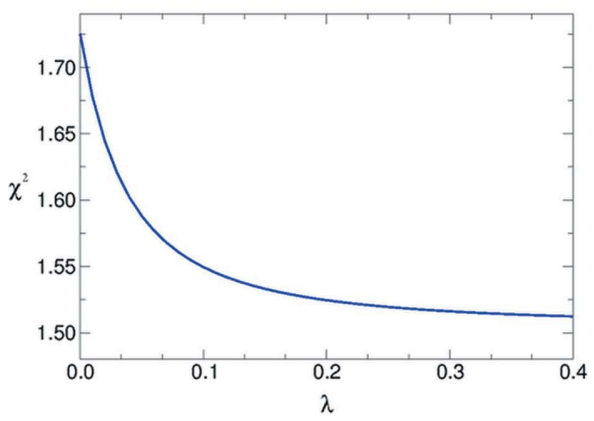

(b)

Figure 3

(a) Chirgwin-Coulson weights $\left(K_{A}, K_{C}\right.$ in blue and $K_{B}$ in red) and $(b) \chi^{2}$ statistical agreement as a function of the external multiplier $\lambda$ for the $\mathrm{XC}$ ELMO-VB calculations performed on the crystallographic structure of naphthalene at $100 \mathrm{~K}$. different degrees of deconvolution of thermal motion.

The effects of the experimental constraints on the Chirgwin-Coulson weights are also very clear in Fig. 3(a) where we can better observe how the weights change as a function of the external multiplier $\lambda$ for the crystallographic structure determined at $100 \mathrm{~K}$. The variation of the $\chi^{2}$ statistical agreement is also shown in Fig. 3(b). Analogous trends have also been observed for the crystallographic structures Figs. S2-S4 in the supporting information).

For the sake of completeness, we want to point out that, not considering individually the weights of the single resonance forms, the delocalized picture of the electronic structure of naphthalene jointly provided by the doubly degenerate resonance forms $A$ and $C$ predominates over the description given by resonance structure $B$, both in the gas (unconstrained calculations) and crystalline (X-ray constrained calculations) phases. This is in agreement with recent studies conducted by Gatti, Lo Presti and co-workers (Monza et al., 2011; Gatti et $a l ., 2016)$ that exploited the Source Function descriptor to analyse the delocalization pattern of naphthalene by considering also the electron-density distribution obtained from the multipole model refinement of the experimental data used in the present paper (Oddershede \& Larsen, 2004).

\section{Conclusions}

In this short communication we have presented a firstprototype multi-determinant X-ray constrained wavefunction approach that mainly exploits pre-determined ELMO single Slater determinants to expand a valence-bond-like wavefunction. The method can be particularly useful to study molecules with a multi-reference character and, consequently, to directly extract the weights of the resonance structures of the system under examination from experimental structure factors. Test calculations performed by exploiting highresolution X-ray diffraction data for naphthalene have shown that the relative importance of resonance structures may change when the resonance weights are determined by also considering experimental structure factors instead of simply minimizing the energy of the system. The promising preliminary results obtained with the current version of the technique encourage us to further improve the method in the near future, in particular by also introducing an optimization determined at the other temperatures $(135,170$ and $205 \mathrm{~K}$; see 
of the ELMO Slater determinants used to expand the multideterminant wavefunction.

\section{Acknowledgements}

The author gratefully acknowledges Anders $\varnothing$. Madsen, Jette Oddershede and Sine Larsen for providing the experimental $\mathrm{X}$-ray diffraction data (cif and hkl files) associated with the structures of naphthalene at the different temperatures.

\section{References}

Boehme, R. F. \& La Placa, S. J. (1987). Phys. Rev. Lett. 59, 985-987. Bučinský, L., Jayatilaka, D. \& Grabowsky, S. (2016). J. Phys. Chem. A, 120, 6650-6669.

Bytheway, I., Grimwood, D. J., Figgis, B. N., Chandler, G. S. \& Jayatilaka, D. (2002). Acta Cryst. A58, 244-251.

Bytheway, I., Grimwood, D. J. \& Jayatilaka, D. (2002). Acta Cryst. A58, 232-243.

Chirgwin, B. H. \& Coulson, C. A. (1950). Proc. R. Soc. London Ser. A, 201, 196-209.

Clinton, W. L., Frishberg, L. J., Massa, L. J. \& Oldfield, P. A. (1973). Int. J. Quantum Chem. 7, 505-514.

Clinton, W. L., Galli, A. J., Henderson, G. A., Lamers, G. B., Massa, L. J. \& Zarur, J. (1969). Phys. Rev. 177, 27-33.

Clinton, W. L., Galli, A. J. \& Massa, L. J. (1969). Phys. Rev. 177, 7-13.

Clinton, W. L., Henderson, G. A. \& Prestia, J. V. (1969). Phys. Rev. 177, 13-18.

Clinton, W. L. \& Lamers, G. B. (1969). Phys. Rev. 177, 19-27.

Clinton, W. L. \& Massa, L. J. (1972a). Int. J. Quantum Chem. 6, 519523.

Clinton, W. L. \& Massa, L. J. (1972b). Phys. Rev. Lett. 29, 1363-1366.

Clinton, W. L., Nakhleh, J. \& Wunderlich, F. (1969). Phys. Rev. 177, $1-6$.

Dos Santos, L. H. R., Genoni, A. \& Macchi, P. (2014). Acta Cryst. A70, 532-551.

Frishberg, C. A. \& Massa, L. J. (1978). Int. J. Quantum Chem. 13, 801810.
Frishberg, C. A. \& Massa, L. J. (1981). Phys. Rev. B, 24, 7018-7024. Frishberg, C. A. \& Massa, L. J. (1982). Acta Cryst. A38, 93-98.

Gatti, C., Saleh, G. \& Lo Presti, L. (2016). Acta Cryst. B72, 180-193. Genoni, A. (2013a). J. Phys. Chem. Lett. 4, 1093-1099.

Genoni, A. (2013b). J. Chem. Theory Comput. 9, 3004-3019.

Genoni, A., Dos Santos, L. H. R., Meyer, B. \& Macchi, P. (2017). IUCrJ, 4, 136-146.

Genoni, A. \& Meyer, B. (2016). Adv. Quantum Chem. 73, 333-362.

Gillet, J.-M. (2007). Acta Cryst. A63, 234-238.

Gillet, J.-M. \& Becker, P. J. (2004). J. Phys. Chem. Solids, 65, 2017 2023.

Gillet, J.-M., Becker, P. J. \& Cortona, P. (2001). Phys. Rev. B, 63, 235115.

Goldberg, M. J. \& Massa, L. J. (1983). Int. J. Quantum Chem. 24, $113-$ 126.

Grabowsky, S., Genoni, A. \& Bürgi, H.-B. (2017). Chem. Sci. doi: 10.1039/c6sc05504d.

Grimwood, D. J., Bytheway, I. \& Jayatilaka, D. (2003). J. Comput. Chem. 24, 470-483.

Grimwood, D. J. \& Jayatilaka, D. (2001). Acta Cryst. A57, 87-100.

Hudák, M., Jayatilaka, D., Perašínová, L., Biskupič, S., Kož̌̌šek, J. \& Bučinský, L. (2010). Acta Cryst. A66, 78-92.

Jayatilaka, D. (1998). Phys. Rev. Lett. 80, 798-801.

Jayatilaka, D. (2012). Modern Charge-Density Analysis, edited by C. Gatti \& P. Macchi, pp. 213-257. Dordrecht: Springer.

Jayatilaka, D. \& Grimwood, D. J. (2001). Acta Cryst. A57, 76-86.

Massa, L., Goldberg, M., Frishberg, C., Boehme, R. F. \& La Placa, S. J. (1985). Phys. Rev. Lett. 55, 622-625.

Monza, E., Gatti, C., Lo Presti, L. \& Ortoleva, E. (2011). J. Phys. Chem. A, 115, 12864-12878.

Oddershede, J. \& Larsen, S. (2004). J. Phys. Chem. A, 108, 1057-1063.

Schmider, H., Smith, V. H. Jr \& Weyrich, W. (1990). Trans. Am. Crystallogr. Assoc. 26, 125-140.

Schmider, H., Smith, V. H. Jr \& Weyrich, W. (1992). J. Chem. Phys. 96, 8986-8994.

Sironi, M., Genoni, A., Civera, M., Pieraccini, S. \& Ghitti, M. (2007). Theor. Chem. Acc. 117, 685-698.

Stoll, H., Wagenblast, G. \& Preuss, H. (1980). Theor. Chim. Acta, 57, 169-178.

Weyrich, W. (2006). Lect. Ser. Comput. Sci. 5, 1-3. 